\title{
Analysis on Damages of Network Alienation on Mental Health of Students and Countermeasures
}

\author{
Weiqi Gao \\ Liberation Army Benggu Sergeancy Academy, Benggu Anhui, China \\ weiqigao@163.com
}

Keywords: Network information alienation, Students, Mental health

\begin{abstract}
While providing rich learning resource and wide social communication space for students, rapid development of internet technology has negative influence. For example, the network alienation damages the cognition, emotion, self-awareness and personality of students. For network information alienation, we should take a series measures to improve information literacy and competence of using information, enhance network mental health education and prevent from the damage on mental health.

With rapid development of internet technology and the popularity of mobile communication products such as portable computers and tablet computers, the network attracts wide attention of the students with unique glamour. While providing rich learning resource and wide social communication space for students, rapid development of internet technology has negative influence. Especially when the world outlook, outlook on life and values of young students are generating, the students are easy to lose themselves in complicated network world, and there is information alienation, which causes psychological problems.
\end{abstract}

\section{Network Information Alienation and Representation}

Network information alienation means that the subject of network control and utilization are interfered by various factors in the process of achieving and using network information, lose the dominant role and becomes external alienation and are objected by network information. Network information alienation causes a series of psychological problems. [1]

Firstly, excessive dependence. The computer network technology develops rapidly, and there are more and more accesses to internet. The development of new network tools such as netbook, tablet computer and smart phones not only makes information acquisition efficient and convenient, but also makes the tools become the external coercive power to control the users. The dependence of the people on the network is stronger. If they have no tools to access to internet, they may feel isolated from the society, ad the social life may be not normal.

Secondly, blind worship for information. Blind worship for information means the blind attitude and extreme measures to infinitely exaggerate the value and functions of the information based on wrong understanding for the essence of information. Wide application of internet makes information achieve more and more reputations, for example, information is knowledge, and information is wealth. The functions of information is exaggerated infinitely, which makes some people lose the subjectivity in the process of pursing new information.

Thirdly, inexplicable information fear. The development of modern information technology not only increases the chances of people choosing information, but also makes people feel that the mastered information is not enough. There is irreconcilable contradiction between rapid development of social information and lagging information processing capacity of the people, which makes people anxious, and makes people feel fearful in the process of contacting with network.

Lastly, complicated information disorder. Network information lacks of selection and supervision. Lots of junk information not only makes network information environment degraded, but also makes the value system not solid and makes the people without resolving ability confused. 
While facing disordered information, they don't know how to choose, judge and use, and they are hesitated and overwhelmed [2].

\section{Damage of Network Information Alienation for Mental Heath of Students}

Network information alienation weakens self-awareness of students and makes the students lose subjectivity. Being immersed in network world for a long time is harmful to the improvement of self-awareness of the young students. The young students have strong thirst for knowledge, and they are easy to accept the new things. The amass data, beautiful music, lively video and rich games on the internet has great temptation and stimulation for them. While continuously opening network connections, many students lose themselves without consciousness. And some students have powerful psychological dependence on network, and are addicted into the network. Excessive expansion of network information is easy to make the young students with weak resolving ability and self-control lose the subjectivity for value judgment and selection.

Network information alienation makes perceptivity of students fall and makes thought disordered. Psychological researches indicate that contacting a thing for a long time is easy to make the susceptibility of the thing reduce. Frequent surfing the internet, browsing the page for a long time and excessive information input is harmful to the susceptibility of the people, and is impossible to make thought disordered. Acquiring information is very easy in the excessive information world. The problems can be solved quickly without thinking deeply. The brain is inundated with digested information, which influences the thought deepness of the people ad makes the thinking ability of the people fall [3].

Network information alienation makes affect of some students indifferent and makes interpersonal relationship disordered. Network has the feature of tacitness. Once the students are addicted into the information, they may forget their real identity and ignore their role. The behaviors are easy to break away from the supervision of the school and the society, and lack of responsibility. It is easy to make that some students are only willing to seek the joy of life in network. And they are indifferent for the other people, and are not interested in the group activities, which makes the affect indifferent and makes interpersonal relationship disordered.

Network information alienation makes the personality of students distorted. Network creates a virtual space, which makes that the students are not enslaved to various objective existences in the real world, and they can hide their real identities to break away from moral constraints. They can go their own ways in the network. But the problem is that the difference of virtual and real space-time makes that the personality needs to be cracked into dual structure. One structure is appropriate for network world, and the other is suitable for real life. If it can't be transformed timely, the personality may be cracked and distorted, which not only is difficult to correctly estimate the requirements of social environment and army's situation for them, and is difficult to correctly evaluate their behaviors, but also is difficult to make proper reactions to the stimulation of the surroundings [4].

\section{Countermeasures of Mental Health Education for Network Information Alienation in Military Academy}

Educating the students to adjust the subjective and objective role of the people and information, and cultivating good self-awareness. People is the subject of network and information, network information serves people, and the development of information activities depends on the subjectivity of people. We should make the students fully realize that they are the deciders of network life and the subjects of information activities. The purpose of browsing network and downloading information is to serve their learning, training and life rather than being controlled by the network, which is helpful for the students to set up the self-esteem, self-confidence and self-improvement concept. In addition, we should improve the ability of the students recognizing information and adjusting themselves, which makes them determine their value orientation, idea and faith. 
Enhancing information quality education of students and improving ability of using information. Information society and the information war in the future not only enriches the connotation of information quality of the soldiers, but also proposes new requirements for the quality of the students. Information quality not only is an important index to evaluate the comprehensive quality of the soldiers, but also is the survival skill to process and use information, improve operational effectiveness and realize success in the information war in the future. To make the students break away from the control of information alienation needs to cultivate and improve information quality of students to make them master necessary information knowledge and posses good information processing ability, Only improving information quality of students can make the students develop independent learning attitude and scientific learning method, and have the consciousness and ability to pursue new knowledge and use new information, which is helpful for them to grow up healthily in information society and information revolution in the army.

Emphasizing personality education of students and improving consciousness of students for behaviors. Personality is internal tendency of individuals for behaviors. It is the integration of the individuals for ability, quality, character, requirements, motivation and value view while adapting to environment, and is the psychological feature of individuals in socialization process. [5] Good personality has great significance for mental health and development of individuals. In network era, the personality of students enhances. Enhancing personality education on students is good for them to take positive attitude for lots of network information and overcome various adverse factors.

Developing network mental health education. We should appropriately pay attention to the psychological status of the students, and develop mental education. According to their psychological characteristics, we should investigate the negative influences of network on psychology of different students, and formulate feasible psychological education schemes. And we should set up relevant psychology course, enrich spare-time culture life of students, and implement mental health education. With the coming of information age, there are higher and higher requirements for psychological quality of students, so we need to enhance the mental defense ability of students for network information alienation.

\section{References}

[1] F.G. Huo and S.X. Liu, Theory of information moral education-research on information quality and ideological and political education information of university students, Beijing: People's Publishing House, 2008.

[2] D. Li, L.R. Zeng and Y.M. Huang, Analysis of lose and reconstruction of subjectivity of network morality for college students, Lanzhou Academic Journal, 2004(2):198—200.

[3] J.Z. Wang. Research on influence of network culture on mental health of people, Education Exploration, 2005(4):92-94.

[4] C.Q. Tong, G.F. Ye and Q. Yang. Influence of network on psychology of college students and education measures, Journal of Qinghai Normal University (Natural Science), 2002(4):148—151.

[5] X.T. Huang, Personality Psychology, Zhejiang Education Press, 2002,9. 